\title{
ESTUDIO COMPARATIVO EN LA RESPUESTA INMUNE HUMORAL DE IgM E IgG EN CERDO CRIOLLO MEXICANO Y COMERCIAL
}

\author{
IgM AND IgG IMMUNE RESPONSE IN MEXICAN CREOLE AND COMERCIAL PIGS: \\ ACOMPARATIVESTUDY
}

\author{
Mejía-Martínez, K. ${ }^{1 A}$, Lemus-Flores, C. ${ }^{1 *}$ y Zambrano-Zaragoza, J.F. ${ }^{1}$ \\ ${ }^{1}$ Universidad Autónoma de Nayarit. Ciudad de la Cultura Amado Nervo s/n. C.P. 63190 Tepic Nayarit. Mexico. \\ Akarinamej@hotmail.com; *clemus@nayar.uan.mx
}

\section{Palabras ClaVe ADICIONALES}

Respuesta humoral. Cerdos Criollos mexicanos. Resistencia.

\section{RESUMEN}

En el presente estudio, se analizó la respuesta humoral $\lg \mathrm{G}$ e IgM en cerdos Criollos mexicanos (Cuino (CC) y Pelón Mexicano (CPM)) y se comparó con la de cerdos comerciales (CCO) F1 Yorkshire-Landrace, para establecer diferencias que nos permitieran utilizarlos como indicador de resistencia a la enfermedad. Para ello, se analizaron 76 animales procedentes de la Unidad Académica de Medicina Veterinaria y Zootecnia de la Universidad Autónoma de Nayarit. Cerdos destetados de 45 días de edad fueron retados con la vacuna bacterina mixta porcina, que contiene cepas de Salmonella, Escherichia coli y Pasteurella y se evaluaron los niveles de anticuerpos por ELISA, utilizando la misma mezcla antigénica sonicada para sensibilizar las placas. Se tomaron muestras de sangre a los 8 y 15 días posteriores al reto, para evaluar la respuesta de IgM e IgG respectivamente. Se encontró que los niveles de IgM anti-bacterina mixta porcina fueron más altos en el CPM que en las otras razas analizadas $(p<0,05)$. En el caso de los niveles de $\mathrm{lg} G$, no se encontraron diferencias en los niveles de anticuerpos IgG anti-bacterina mixta porcina entre el CC y el CPM, sin embargo, ambas razas criollas fueron diferentes que la CCO $(p<0,05)$. Se observó que los CCO tienen menor respuesta de anticuerpos IgG. Los resultados encontrados sugieren que los CPM y en menor medida los CC tienen una mejor respuesta humoral contra Salmonella, Escherichia coli y Pasteurella que los CCO.

Recibido: 6-2-08. Aceptado: 17-9-08.

\section{ADDITIONAL KEYWORDS}

Humoral response. Mexican Creole pigs. Resistance.

\section{SUMMARY}

The aim of this work was to compare the humoral IgG and IgM immune response between Mexican Creole, Cuino (CC) and Mexican Pelon (CPM) pigs and commercial F1 Yorshire-Landrace pigs (CCO) to use it as indicators for resistance to disease. Seventy six animals obtained from Unidad Académica de Ciencias Veterinarias y Zootécnicas from Universidad Autónoma de Nayarit were included. After shoot, all animals were challenged with bacterina mixta porcina, which included Salmonella, Escherichia coli and Pasteurella antigens and the antibody levels were evaluated at eight and fifteen days after by ELISA. IgM anti-bacterina mixta porcina were significantly higher in $\mathrm{CPM}$ than in $\mathrm{CC}$ and $\mathrm{CCO}(\mathrm{p}<0.05)$. For IgG levels, statistical differences were found neither between CPM and CC nor between $\mathrm{CC}$ and $\mathrm{CCO}$. However, CPM showed higher levels than CCO. The results strongly suggest that CPM and CC have better humoral immune response than $\mathrm{CCO}$ pigs.

\section{INTRODUCCIÓN}

Un alto porcentaje de las razas de cerdos domésticas que proveen de alimento a la humanidad están en peligro de extinción. Sin embargo, las razas de cerdos indígenas poseen comúnmente rasgos valiosos, como adaptación a condiciones difíciles, incluyendo tolerancia a enfermedades parasita- 


\section{MEJÍA-MARTÍNEZ, LEMUS-FLORESYZAMBRANO-ZARAGOZA}

rias e infecciosas, sequía y pobre calidad alimenticia(CVID, 2003; Sierra et al., 2003), que los hacen de interés para el establecimiento de características que permitan mejorar en un futuro las especies animales. En este contexto, el cerdo Criollo Mexicano, podría ser un espécimen fructífero para la investigación por muchos años.

Recientemente en México se ha impulsado el estudio y caracterización de los recursos genéticos y moleculares de estas especies, toda vez que se ha comprendido la relevancia de las variantes genéticas tan extensas, pero tan pobremente caracterizadas, más aún cuando se trata de poblaciones criollas o nativas (Mariscal, 1998).

El cerdo Criollo Mexicano (CCM) fue reportado por la FAO como un cerdo en peligro de extinción (FAO, 2000); es una especie poco valorada, que se cree fue introducida en México en tiempos de la conquista y que de forma natural ha sobrevivido a distintas condiciones ecológicas incluyendo factores infecciosos y limitaciones nutricionales. Es fuente de gran diversidad biológica, además de suponerse que tiene alta resistencia a enfermedades (Flores, 1992; Lemus et al., 2003).

La gran importancia agropecuaria del cerdo ha propiciado un notable incremento de las investigaciones dedicadas al sistema inmunológico de este animal. Sin embargo, no se podrá contribuir a una reducción de las enormes pérdidas económicas, sin un mejor conocimiento del sistema inmunológico porcino (Saalmüller, 1998).

Los anticuerpos son parte de la rama humoral de la respuesta inmunológica. Éstos son producidos por linfocitos B en respuesta a un estímulo antigénico y representan uno de los mecanismos bactericidas más importantes en el organismo. En el cerdo se han descrito cuatro isotipos de anticuerpos, IgM, IgG, IgA e IgE, aunque no se ha descartado la existencia de IgD porcina, ya que los estudios no ha sido concluyentes (SánchezVizcaíno, 2004).

La respuesta de anticuerpos puede divi- dirse en respuesta primaria y secundaria. La respuesta primaria, caracterizada por la producción de anticuerpos de clase $\operatorname{IgM}$, de 5 a 14 días después del reto antigénico. Los anticuerpos producidos son de baja afinidad, aunque no así su avidez, ya que son moléculas pentaméricas. Dentro de las funciones biológicas de esta clase de anticuerpos, esta la de activar complemento, neutralización viral y actividad bactericida (Abbas y Lichtman, 2004).

La respuesta secundaria se caracteriza por la producción de otros isotipos de anticuerpos, principalmente $\mathrm{IgG}$, que es el de mayor concentración en el cerdo. Aparece después de 15 días del reto, sus niveles séricos aumentan rápidamente, tiene un mayor tiempo de vida media, y en esta respuesta se genera memoria inmunológica. La afinidad de los anticuerpos es mayor, aunque su avidez menor que la de IgM. IgG tiene múltiples funciones biológicas, entre las que se encuentran la actividad bactericida, antiviral, citotóxica y la opsonización de microorganismos (Tizard, 2002).

Debido a la presión de selección intensa que se realiza actualmente en las razas de cerdos con alta explotación comercial, se ha propiciado que disminuyan notablemente su variabilidad genética; esto ha ocasionado la fijación de mutaciones genéticas indeseables, así como la disminución de resistencia a enfermedades (Fujii et al., 1991; O'Brien et al., 1993).

En algunos estudios moleculares, se ha demostrado que los cerdos criollos tienen mayor heterocigosidad, por lo que esto pudiera ser determinante de una posible resistencia a enfermedades (Lemus et al., 2001; Canul et al., 2005; Martínez et al., 2000; Martínez et al., 2005).

En Nayarit, México, como en algunos países de América, se dispone de cerdos criollos que han sobrevivido por más de 500 años sin control sistematizado de producción ni salud, lo que hace suponer que cuentan con una alta resistencia a enfermedades (Benítez y Sánchez, 2001; Lemus y Alonso, 2005). 


\section{RESPUESTA HUMORAL EN CERDO CRIOLLO MEXICANO}

El objetivo de este trabajo fue comparar la respuesta inmunológica humoral en los cerdos Criollos Mexicanos y cerdos comerciales (CCO) F1 Yorkshire-Landrace después de la vacunación, como indicadores de resistencia a enfermedad.

\section{MATERIAL Y MÉTODOS}

\section{Animales}

Se emplearon 76 animales criados procedentes de la Unidad Académica de Medicina Veterinaria y Zootecnia de la Universidad Autónoma de Nayarit, divididos en tres grupos:

25 cerdos Pelón Mexicano (CPM),

25 cerdos Cuino (CC),

26 cerdos de raza comercial (F1 YorshireLandrace) (CCO).

Los cerdos fueron destetados a los 45 días de edad y retados antigénicamente con bacterina mixta porcina (Intervet/ SAGARPA-B-0273-133) que contiene cepas de Salmonella, Escherichia coli y Pasteurella). Todos los cerdos se mantuvieron en condiciones ambientales no controladas durante el experimento.

\section{OBTENCIÓNDE LAS MUESTRAS BIOLÓGICAS}

Se obtuvieron $5 \mathrm{ml}$ de sangre de la vena cava, en tubos sin anticoagulante a los 8 y 15 días después del reto, para determinar los niveles de IgM e IgG respectivamente. El suero se obtuvo por centrifugación a 1500 rpm durante 15 minutos y se guardó en alícuotas a $-20^{\circ} \mathrm{C}$ hasta su uso.

\section{AntígenO}

Cinco mililitros de la bacterina mixta porcina se sonicaron ( 30 ciclos de 30 segundos a intervalos de 1 minuto) y se determinó la concentración de proteínas por el método de Lowry (Lowry et al., 1951). Esta mezcla antigénica fue guardada en alícuotas a $-20^{\circ} \mathrm{C}$.

DETERMINACIÓN DE LOS NIVELES DE IGGE IGM ANTIBACTERINAMIXTA PORCINA

Se sensibilizaron placas de poliestireno de 96 pozos de fondo plano (Corning), con $1 \mu \mathrm{g}$ del antígeno/pozo, diluido en regulador de carbonatos $\mathrm{pH}$ 9,6 y se incubó toda la noche a $4^{\circ} \mathrm{C}$. Posteriormente, la placa se lavó tres veces con PBS-Tween al 0,05\% (PBS-T) y se bloqueó con leche descremada al 5\% diluida en PBS-leche (PBS-L) durante una hora a $37^{\circ} \mathrm{C}$. Después del bloqueo, se repitió el proceso de lavado y se adicionó el suero problema diluido 1:100 en PBS-L y se incubó una hora a $37^{\circ} \mathrm{C}$. Transcurrido el tiempo de incubación se repitió el proceso de lavado y se adicionó el anticuerpo conjugado, IgG de cabra anti-IgM o anti-IgG de cerdo, conjugado a peroxidasa (RocklandR) diluido 1:5000 en regulador de bloqueo y se incubó una hora a $37^{\circ} \mathrm{C}$. Enseguida se repitió el proceso de lavado y se reveló la interacción con o-fenilendiamina $(0,2 \mathrm{mg} /$ $\mathrm{ml}$ ) y $0,012 \%$ de $\mathrm{H}_{2} \mathrm{O}_{2}$ en regulador citratofosfato (ácido cítrico al $0,1 \mathrm{M}$ y $\mathrm{Na}_{2} \mathrm{HPO}_{4}$ al $0,2 \mathrm{M}) \mathrm{pH} \mathrm{5,0}$ y se incubó a temperatura ambiente y en oscuridad durante 25 minutos para IgM y 15 minutos para IgG. La reacción se detuvo adicionando $50 \mu \mathrm{lde} \mathrm{H}_{2} \mathrm{SO}_{4} 8 \mathrm{~N}$. Se leyó la absorbancia a $490 \mathrm{~nm}$ en un lector de microplacas de ELISA(Bio-Rad modelo 680). En todos los casos se restó la absorbancia obtenida de los controles sin antígeno.

\section{AnÁlISISESTADÍSTICO}

Se utilizó la prueba de Kruskal-Wallis para comparar los niveles de IgM e IgG antibacterina mixta porcina en las diferentes razas de cerdos y la prueba de Wilcoxon, para conocer las diferencias entre los distintos pares de comparaciones raciales. En todos los casos se utilizó el software SAS (2002).

\section{RESULTADOSYDISCUSIÓN}

Al muestreo de los cerdos ocho días después de la vacunación, todos los lechones de las tres razas presentaron niveles de IgM anti-bacterina mixta porcina, sin diferentes estadísticamente significativas en el $\operatorname{CCO}(0,341 \pm 0,070)$ yCC $(0,336 \pm 0,065)$; mien- 
tras que la raza $\mathrm{CPM}(0,426 \pm 0,102)$ mostró niveles de IgM más elevados con diferencias estadísticas significativas $(\mathrm{p}<0,05)$ (figura 1).

En el caso de los niveles de IgG antibacterina mixta porcina, se encontró que los cerdos de las tres razas presentaron niveles de esta inmunoglobulina, siendo la raza comercial la que tuviera niveles significativamente menores $(0,220 \pm 0,049)$ que los de las razas criollas $(\mathrm{p}<0,05)$. Los niveles de IgG en la raza $\mathrm{CPM}(0,604 \pm 0,358)$ y $\mathrm{CC}$ $(0,473 \pm 0,302)$ no mostraron diferencias estadísticamente significativas (figura 2).

Brown et al. (2006) realizaron un trabajo con la finalidad de evaluar las características morfológicas intestinales y el desarrollo del sistema inmune de cerdos comerciales de las razas Yorkshire, Landrace y Duroc de 7, 14 y 18 días de nacidos, midiendo entre otras variables, la concentración de inmunoglobulinas de tipo $\operatorname{IgM}$, no encontraron diferencias significativas en los valores obtenidos de esta inmunoglobulina entre razas; no obstante se reportan cifras de $\operatorname{IgM}$ en suero de $1,121 \mathrm{ng} / \mathrm{ml}$ a los 7 días, 966 y $1,154 \mathrm{ng} / \mathrm{ml}$ a los 14 y 18 días de nacidos respectivamente; observando una pequeña disminución y después un aumento en la concentración de IgM a los 18 días de nacidos, por análisis complementarios llegaron a la conclusión que el sistema inmune porcino va cambiando conforme va declinando la inmunidad pasiva, estos cambios son muy importantes en la sobrevivencia de los cerdos ya que se hace notar que en siguientes retos antigénicos va madurando el sistema inmunológico del cerdo, mientras la protección por la inmunidad maternal pasiva declina, efecto que hace a los animales más resistentes, de acuerdo con esto es probable que los CPM maduren su sistema más pronto. Carrón et al. (2005), analizaron las respuesta inmunitaria humoral en cerdos (de 2 y 4 meses de edad) de raza ibérica infectados experimentalmente por un parásito, observaron una clara diferencia entre la respuesta humoral de $\operatorname{IgM}$ en ambos lotes

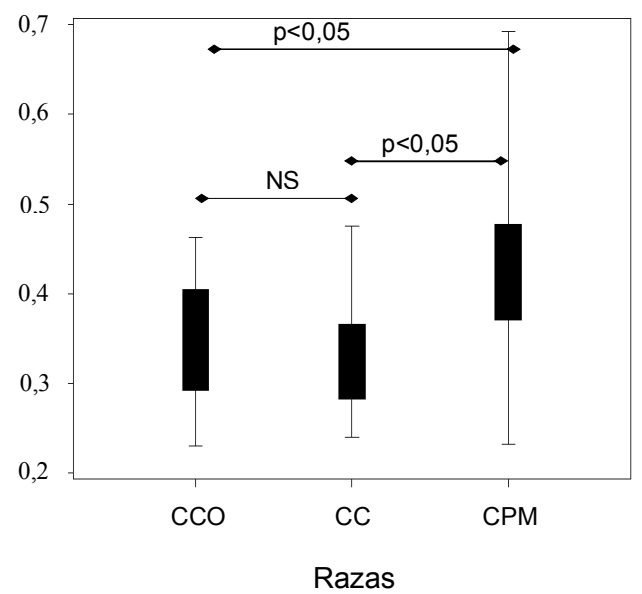

Figura 1. Niveles de anticuerpos IgM antibacterina mixta porcina a los 8 días después de la inmunización en las diferentes razas de cerdo. CCO (cerdo comercial), CC (cerdo Cuino), CPM (cerdo Pelón Mexicano). La absorbancia fue determinada a $492 \mathrm{~nm}$. (IgM to bacterina-mixta porcina levels eight days after challenge in different pigs breed. CCO (commercial pigs), CC (Cuine pigs), CPM (Mexican Pelon pigs). Absorbance was determined at $490 \mathrm{~nm}$ ).

de edad, en los de mayor edad no hubo evolución temporal, en cambio en los animales jóvenes se presentaron evoluciones significativas desde los 7 días después de la vacunación, estos datos coinciden con nuestro estudio ya que se observó el incremento de los niveles de $\operatorname{IgM}$ a los 8 días después de la vacunación.

Las concentraciones de $\operatorname{IgM}$ en cerdos de mayor edad (después de 8 semanas de edad) no varían de forma marcada, ya que después de ese tiempo se reporta que los cerdos han alcanzado ya su maduración inmunológica (Buxadé, 1996).

Como puede observarse, las tres razas de cerdo analizadas responden con anticuerpos de clase IgMe IgGal ser inmunizados con la vacuna. Sin embargo, la raza CPM presenta mayores niveles de anticuerpos tipo IgM que las razas $\mathrm{CCO}$ y CC. Esto puede deberse a que han obtenido mayores concentracio- 


\section{RESPUESTA HUMORAL EN CERDO CRIOLLO MEXICANO}

nes de inmunoglobulinas tipo IgM a través de la leche materna, ya que se ha demostrado que en la placenta no existe traslado alguno de inmunoglobulinas maternales por circulación fetal (Tlaskalowá-Hogenová et al., 1994); y que es hasta la tercera semana de vida que el cerdo comienza la propia producción de anticuerpos y sólo hasta la sexta u octava es cuando completa su maduración inmunológica (Buxadé, 1996).

Allen et al. (2000), mencionan en un estudio en cerdos que la estructura histológica de la placenta no permite la transferencia al útero de inmunoglobulinas, siendo la única fuente de transferencia de ellas el amamantamiento, lo que le permitirá al lechón que mientras alcanza su madurez inmunológica pueda ser protegido por los anticuerpos de la madre, factor que se considera que en este estudio pudo influir y que las inmunoglobulinas tipo $\mathrm{M}$ medidas pue-

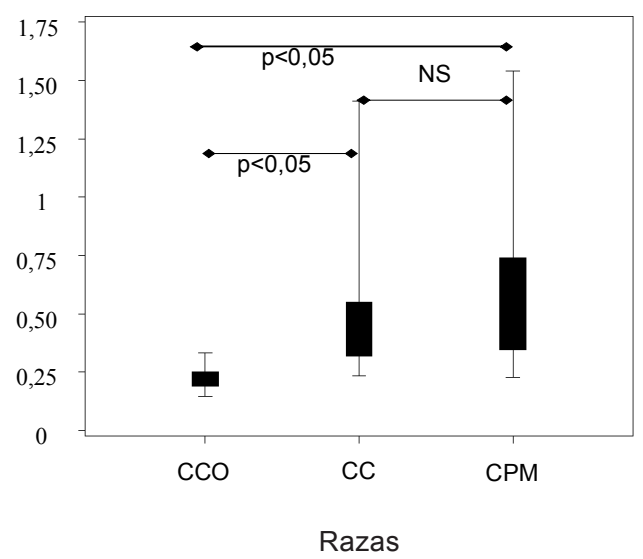

Figura 2. Niveles de anticuerpos $\operatorname{Ig} G$ antibacterina mixta porcina a los 15 días después de la inmunización en las diferentes razas de cerdo. CCO (cerdo comercial), CC (cerdo Cuino), CPM (cerdo Pelón Mexicano). La absorbancia fue determinada a 492 $n m$. (IgG to bacterina-mixta porcina levels fifteen days after challenge in different pigs breed. CCO (commercial pigs), CC (Cuine pigs), CPM (Mexican Pelon pigs). Absorbance was determined at 490 $\mathrm{nm})$. den ser producto de los anticuerpos de la madre, sumado a la respuesta inmunológica de los lechones. En estudios inmunológicos realizados con cerdos de líneas comerciales se conoce que el lechón al nacimiento, se encuentra prácticamente desprovisto de anticuerpos, esto es debido a las seis capas de tejido que contiene la placenta del cerdo, por lo que la inmunidad pasiva es adquirida por medio del calostro al ingerir anticuerpos maternales (Newby et al., 1982).

Las tres poblaciones raciales de este estudio estuvieron en el mismo ambiente y manejo técnico, por lo que es de suponer que si tuvieron contacto previo con las bacterias empleadas en la inmunización, el efecto fue similar en las tres razas, es por esto que las diferencias se pueden atribuir a la raza.

Para el caso de IgG se observó que todas las razas responden con esta clase de anticuerpos, aunque no se encontró diferencia en los niveles de esta clase de anticuerpos entre las razas criollas, CPM y CC. Para el caso de la raza CCO se encontró que presentan menores niveles que las otras dos razas analizadas. Esto es relevante, ya que los anticuerpos de clase IgG son los principales en la respuesta secundaria, que además asegura que hay memoria inmunológica, con lo que los cerdos estarían en mejores condiciones de respuesta hacia los patógenos. El que presenten niveles mayores podría indicar que tienen un mayor número de clonas con receptores para antígenos de las bacterias analizadas, lo que nuevamente redundaría en una mejor protección.

Aunque existen escasos estudios realizados con la finalidad de medir la respuesta inmune en cerdos criollos, Guerrero et al. (2008a) midieron la respuesta inmune celular en CPM comparándola con la de los cerdos comerciales; los resultados mostraron una respuesta similar en la linfoproliferación y fagocitosis en ambos genotipos de cerdos, antes y después de la etapa del destete. Sin embargo, existió una tendencia 


\section{MEJÍA-MARTÍNEZ, LEMUS-FLORESYZAMBRANO-ZARAGOZA}

de dar una mayor respuesta inmune celular en los CPM que en los CCO. Lo que hace considerar que la respuesta inmune celular del CPM tiende a ser mejor que la de un cerdo comercial mejorado, como el Yorkshire x Landrace, debido quizá a su memoria inmunológica, rusticidad, a su mejor adaptación al medio y a que pueden ser reservorios de determinantes genéticos de resistencia natural a diferentes enfermedades.

Esto es impostante en cerdos nativos ya que la inmunidad innata puede controlar la infección hasta que la respuesta inmune adaptativa tome el control, diferencía entre lo propio y lo no propio perfectamente. Asimismo, la inmunidad innata es esencial para la inducción y dirección de la respuesta inmune adaptativa (Janeway, 2000). Este sistema es un requisito previo para una eficaz inmunidad adaptativa (Fox y Harrison, 2000). Al contacto con la mayoría de microorganismos, se activa el reconocimiento al patógeno producto de un juego de receptores.

Otro estudio realizado por Guerrero et al. (2008b) con la finalidad de comparar la capacidad de respuesta inmunológica por medio de los niveles de IgG en cerdos CPM y $\mathrm{CCO}$, en cuatro etapas diferentes de maduración inmunológica $(28,32,45$ días de edad sin reto inmunológico y 60 días de edad de los cerdos después del reto inmunológico), se observaron resultados similares a este estudio; los niveles de IgG en los CPM fueron mayores en las diferentes etapas de medición. Por lo que se puede inferir que la respuesta inmune del CPM tiende a ser mayor que la del CCO, debido quizá a su memoria inmunológica, rusticidad y a su mejor adaptación al medio.

Con respecto a la variación genética en las poblaciones criollas de México, se ha reportado que es mayor que en las poblaciones de raza comercial, lo que puede ser un factor a considerar para el desarrollo de una respuesta inmunológica, o bien, esta variabilidad genética propiciar una mejor res- puesta inmune. Los cerdos criollos locales filogenéticamente se encuentran separados genéticamente de los cerdos modernos, situación que sugiere que así se han conservado a pesar de la falta de programas sistematizados de mejora genética (Martínez et al., 2000; Lemus et al., 2001).

En nuestro país no se han aplicado programas de selección productiva para este tipo de cerdos criollos; sin embargo, han sobrevivido por más de 500 años a problemas de zoonosis, lo que hace suponer que tienen alta resistencia a enfermedades, además de representar un reservorio genético para obtener variedades nacionales mejor adaptadas (Benítez y Sánchez, 2001; Lemus et al., 2001; Sierra et al., 2005; Canul et al., 2005).

\section{CONCLUSIONES}

En las tres razas de cerdo se encontraron valores de IgM e IgG antibacterina mixta porcina después de la vacunación.

No se encontraron diferencias significativas ( $p>0,05$ ) entre las razas $\mathrm{CCO}$ y CC para los niveles de $\operatorname{IgM}$, resultando diferente $(\mathrm{p}<0,05)$ y con más altos niveles la raza CPM, ya que los niveles de absorbancia fueron: CCO 0,341 $\pm 0,070$ y CC 0,336 $\pm 0,065 \mathrm{y}$ para CPM 0,426 $\pm 0,102$.

En la determinación de los niveles de IgG después de la vacunación no se encontraron diferencias significativas entre las razas CC y CPM ( $p>0,05)$, resultando diferente y con menores niveles la raza $\mathrm{CCO}(\mathrm{p}<0,05)$; siendo los valores de CCO 0,220 $\pm 0,049$, mientras que CPM 0,604 $\pm 0,358$ y CC 0,473 $\pm 0,302$.

Al estar madres como crías de las tres razas comparadas en el mismo ambiente y no haber vía placentaria transferencia de inmunoglobulinas, se supone entonces que hay un efecto de la raza sin descartar la influencia materna por transmisión de inmunoglobulinas por leche en el amamantamiento, encontrándose que los CPM presentan mejor respuesta inmunológica seguida por CC. 


\section{RESPUESTA HUMORAL EN CERDO CRIOLLO MEXICANO}

\section{AGRADECIMIENTOS}

Los autores agradecen a SAGARPA-

\section{BIBLIOGRAFÍA}

Abbas, K.A. y Lichtman, H.A. 2004. Inmunología celular y molecular. $5^{\text {a }}$ Ed. Editorial GEA Consultoría Editorial. SAUNDERS. Elsevier Science and Elsevier Imprim. Madrid. España. $556 \mathrm{pp}$.

Allen, W.E., Kyoung-Jin, Y. and Zimmerman, J.J. 2000. Immune components in porcine mammary secretions. Viral Immunol., 13: 383-397.

Benítez, O.W. y Sánchez, D.M. 2001. Los cerdos criollos en América Latina. En: FAO (ed.) Los cerdos locales en los sistemas tradicionales de producción. Estudio FAO Producción y Sanidad Animal, 148: 13-35.

Buxadé, C.C. 1996. Zootecnia. Bases de producción animal. Tomo VI. Editorial Mundi Prensa. Barcelona. España. 175 pp.

Brown, C.D., Maxwell, V.C., Erf, F.G., Davis, E.M., Singh, S. and Johnson, B.Z. 2006. Ontogeny of $T$ lymphocytes and intestinal morphological characteristics in neonatal pigs at different ages in the postnatal period. J. Anim. Sci., 84: 567-578.

Canul, S.M., Sierra, V.A., Martínez, M.A., Ortiz, O.J., Delgado, J.V., Vega-Pla, J.L. y Pérez, G.F 2005. Caracterización genética del cerdo Pelón Mexicano mediante marcadores moleculares. Arch. Zootec., 54: 267-272.

Carrón, A., Frontera, E., Sánchez, J., Alcalde, M. y Serrano, F. 2005. Respuesta inmunutaria humoral en cerdos infectados experimentalmente por Ascaris suum. Congreso Internacional Virtual 1-5. http://www.congresocbta. unam.mx/MV13.htm (20/05/2008).

CVID. 2003. Centro Virtual, Investigación y Desarrollo. http://lead/virtualcenter.org/es/dec/ toolbox/Index.htm (30/01/2008).

FAO. 2000. Domestic animal diversity information system: FAO, Rome. http://www.fao.org7dadis7. (diciembre/2002).

Fox, A. and Harrison, L.C. 2000. Innate immunity and graft rejection. Inmunol. Rev., 173: 141-147.

Flores, M.J. 1992. Cría y explotación, enfermedades e industrialización. En: Enciclopedia técnica del ganado porcino. Vol. I. Edit. Limusa. México. D.F. 278 pp.
CONACYT, con número 1472-2002, por la financiación de este proyecto.

Fujii, J., Otsu, K., Zorzato, F., De leon, S., Khanna V.K., Weiler, J.E., O'Brien, P.J. and MacLennan, D.H. 1991. Identification of mutation in the porcine ryanodine receptor that is associated with malignant hyperthermia. Science, 253: 448451.

Guerrero, Q.L.A., Villagómez, D.A.F., Zaitzeva, G., Lemus, C., Taylor, J.J., Galindo, J., Sánchez, D.R., Ayala, M.A., Merlos, M.T. y Roa, J.J. 2008a. Respuesta inmune celular mediante técnicas de linfoproliferación y fagocitosis en cerdos Pelón Mexicano antes y después del destete. Rev. Comput. Prod. Porc., 15: 76-81.

Guerrero, Q.L.A., Villagómez, D.A.F., Huerta, M., Estrada, J., Luquín de Anda, S., Rosales, S.A., Lemus, C., Taylor, J.J., Galindo, J., Sánchez, D.R., Ayala, M.A., Merlos, M.T. y Roa, J.J. 2008b. Comparación de los niveles de IgG en cerdos Pelón Mexicano y Yorkshire $x$ Landrace en diferentes etapas de maduración inmunológica. Rev. Comput. Prod. Porc., 15: 72-75.

Janewey Jr., C.A. 2000. The road less traveled by: The role of innate immunity in the adaptive immune response. Immunol. Rev., 173: 539544.

Lemus, F.C., Ulloa-Arvizu, R., Ramoskuri, M., Estrada, F.J. and Alonso, R.A. 2001. Genetic analisis of Mexican hairless pig populations. J. Anim. Sci., 79: 1-6.

Lemus, F.C., Alonso, M.R., Alonso-Spilsbury, M. and Ramírez, N.R. 2003. Morphologic characteristics in mexican native pigs. Arch. Zootec., 52:197.

Lemus, F.C. y Alonso-Spilsbury, M. 2005. El cerdo Pelón Mexicano y otros cerdos criollos. $1^{\text {a }}$ Edición. Editorial Universitaria. Universidad Autónoma de Nayarit. México. 251 pp.

Lowry, O.H., Rosenbrough, N.J., Farr, A.L. and Randall, R.J. 1951. Protein measurement with the folin phenol reagent. J. Biol. Chem., 193: 355.

Mariscal, A.V. 1998. Impacto del mejoramiento genético sobre la eficiencia reproductiva en cerdos. Tercer Foro de Análisis de los Recursos Genéticos. México, D.F. pp. 92-101. 


\section{MEJÍA-MARTÍNEZ, LEMUS-FLORESYZAMBRANO-ZARAGOZA}

Martínez, A.M., Delgado, J.V., Rodero, A. and Vega-Pla, J.L. 2000. Genetic structure of the Iberian pig breed using microsatellites. Anim. Genet., 31: 295-301.

Martínez, A.M., Pérez-Pineda, E., Vega-Pla, J.L., Barba, C., Velásquez, F.J. y Delgado, J.V. 2005. Caracterización genética del cerdo Criollo Cubano con microsatélites. Arch. Zootec., 54: 369-375.

Newby, T.J., Stokes, C.R. and Bourne, F.J. 1982. Immunological activities of milk. Vet. Immunol. Immunopathol., 3: 67-94.

O'Brien, P.J., Shen, H., Cory, R. and Zhang, X. 1993. Use of a DNA-based test for the mutation associated with porcine stress syndrome (malignant hyperthermia) on 10,000 breeding swine. JAVMA, 203: 842-51.

Saalmüller, A. 1998. Linfocitos T y la respuesta inmune especifica de antígeno contra patógenos varios en el cerdo. Rev. Sci. Tech. Off. Int. Epiz., 17: 71-83.

Sanchez-Vizcaino, J.M. 2004. Curso de introduc- ción a la Inmunología porcina. $2^{\mathrm{a}}$ Edición. Editorial Amer. Laboratorios Hipra. Barcelona, España. http://www.sanidadanimal.info/cursos/ inmuno2/. (20/05/2008).

SAS. 2002. SAS/STAT User's Guide. Release 6.12. Cary NC. SAS Institute, Inc. USA.

Sierra, A., Canal, S.M., Cen, A.F., Rodríguez, C.R., Delgado, J.V. y Martínez, M.A. 2003. El cerdo Pelón Mexicano: programa de conservación genética de una raza en peligro. México. Arch. Zootec., 52: 279-284.

Sierra, A.C., Poot, T.B., Díaz, Z.I., Cordero, A.H. y Delgado, J.V. 2005. El cerdo Pelón Mexicano, una raza en peligro. México. Arch. Zootec., 54: 165-170.

Tizard, I.R. 2002. Inmunología veterinaria. Mc GrawHill Interamericana. México D.F.

Tlaskalová-Hogenová, H., Mandel, L., Trevichavsky, I., Kováru, F., Barot, R. and Sterzl, J. 1994. Development of immune response in early pig ontogeny. Vet. Immunol. Immunopathol., 43: 135-142. 